$$
\text { Arthur Stanloy Pease }
$$

[Reprinted from Appalachia, Vol. XII, No. 3.] 


\section{List of Plants on Three Mile Island. \\ Pteridophyta and Spermatophyta.}

By Arthur Stanley Pease.

This list was prepared as follows : In August, 1903, a manuscript list of plants noted on the island was prepared by A. S. Pease. Specimens were collected of only a few of the more important species. In 1906 specimens were secured by J. H. Emerton (May) and R. A. Ware (July). These specimens of 1906 are deposited in the Club herbarium, as are many others, without memoranda as to date or collector. As such a list as this should be based upon actual evidence of the plants themselves, wherever possible, the manuscript list of 1903 has been cited only when no specimens were to be found in the Club herbarium or that of A. S. Pease. Such reports (indicated in the list by the sign \#) and a few other reports unsubstantiated by evidence should be immediately replaced by actual specimens. In May, 1901, H. P. Kelsey introduced upon the island 481 plants of 62 species. Of these a very few were probably already indigenous there. The others have been entered in the following list, but not numbered, as they are, of course, not a part of the native flora. Some have probably not persisted. The accompanying list is, without doubt, capable of much improvement and increase. The nomenclature is that of the seventh edition of Gray's Manual.

\section{Abbreviations.}

A. Specimens of anonymous collectors with incomplete data. In the A. M. C. herbarium.

E. Plants collected by J. H. Emerton. In the A. M. C. herbarium.

K. Plants introduced by H. P. Kelsey in May, 1901. 
LIST OF PLANTS ON THREE MILE ISLAND. 267

P. Plants collected by A. S. Pease, 24-25 August, 1903. In herbarium of A. S. Pease.

W. Plants collected by R. A. Ware, 9-16 July, 1906. In A. M. C. herbarium.

These plants rest solely upon a MS. list made by A. S. Pease in August, 1903.

I. Polypodiacece.

1. Aspidium cristatum (L.) Sw., var. Clintonianum D. C. Eaton. P., no. 2522.

2. A. marginale (L.) Sw. W.

3. A. spinulosum (O. F. Müller) Sw., var. intermedium (Muhl.) D. C., Eaton. W.

4. A. Thelypteris (I.) Sw. W.

5. Asplenium Filix-femina (L.) Bernh. W.

6. Dicksonia punctilobula (Michx.) Gray. W.

7. Onoclea sensibilis L. A.

8. Phegopteris Dryopteris (L.) Fée. W.

9. P: polypodioides Fée. A.

10. Polypodium vulgare $L$. W.

11. Polystichum acrostichoides (Michx.) Schott. \#

12. Pteris aquilina $\mathrm{L}$. W.

13. Woodsia ilvensis (L.) R. Bs. Miss Adams, May, 1909.

II. Osmundacece.

14. Osmunda cinnamomea L. W.

15. O. regalis L. \#

III. Ophioglossacece.

16. Botrychium obliquum Muhl. An anonymous report of August, 1904.

IV. Lycopodiacece.

17. Lycopodium clavatum L. W.

18. L. complanatum L., var. flabelliforme Fernald. W.

19. L. obscurum L. W.

\section{Taxacex.}

20. Taxus canadensis Marsh. W. Also M. A. Coe, August 6, 1906.

VI. Pinacees.

21. Abies balsamea (L.) Mill. \#

A. Fraseri (Pursh) Poir. K. 
Chamæcyparis thyoides (L.) BSP. K.

22. Juniperus communis L., var. depressa Pursh. A.

J. virginiana L. K.

23. Picea mariana (Mill.) BSP. W. Also K.

Pinus pungens Lamb. $\mathrm{K}$.

24. P. resinosa Ait. W.

25. P. Strobus L. W. Also K.

26. Tsuga canadensis (L.) Carr. W. Also K.

T. caroliniana Engelm. K.

VII. Najadacece.

27. Potamogeton dimorphus Raf. P., no. 2501.

28. P. heterophyllus Schreb. \#

\section{Graminece.}

29. Agropyron caninum (L.) Beauv. var. tenerum (Vasey) Pease \& Moore, f. citation (Scribn. \& Son.) Pease \& Moore. See Rhodora xii. 72. P., no. 2515. Also A.

30. Agrostis alba L. \#

31. A. hyemalis (Walt.) BSP. \#

32. Danthonia spicata (L.) Beauv. W.

33. Muhlenbergia racemosa (Michx.) BSP. P. no. 2526.

34. Oryzopsis asperifolia Michx. E., 22 May, 1906.

35. O. pungens (Torr.) Hitchc. E., 21 May, 1906.

36. Panicum latifolium L. W.

37. Panicum linearifolium Scribn. W.

38. Phleum pratense L. W.

39. Poa compressa L. \#

IX. Cyperacec.

40. Carex brunnescens Poir. P., no. 2516.

41. C. communis Bailey. E., 17 June, 1907.

42. C. fœnea Willd., var. perplexa Bailey. P., no. 3143.

43. C. laxiflora Lam. W. (Possibly one of the varieties of this species.)

44. C. Iupulina Muhl. W. Also P., no. 2520.

45. C. scoparia Schk. \#

46. C. trisperma Dewey. \#

47. Eleocharis tenuis (Willd.) Schultes. \#

X. Eriocaulacec.

48. Eriocaulon septangulare With. M. A. Coe, 12 August, 1906. 


\section{Juncacece.}

49. Juncus pelocarpus Meyer. P., no. 3104.

50. J. tenuis Willd. W.

\section{Liliacece.}

51. Clintonia borealis (Ait.) Raf. A.

Helonias bullata L. $K$.

Lilium Grayi Wats. K.

52. L. philadelphicum L. W.

L. superbum L. K.

53. Maianthemum canadense Desf. W.

54. Medeola virginiana $\mathrm{L}$. W.

55. Oakesia sessilifolia (L.) Wats. \#

56. Polygonatum biflorum (Walt.) Ell. \#

57. Smilacina racemosa (L.) Desf. A.

\section{Iridacece.}

58. Iris versicolor $\mathrm{L}$. W.

59. Sisyrinchium angustifolium Mill. W.

XIV. Orchidacex.

60. Cyripedium acaule Ait. E., 30 May, 1906.

61. C. arietinum R. Br. P., no. 2527. Also E., 23 May, 1906.

62. Epipactis tesselata (Lodd.) A. A. Eaton. A.

63. Habenaria Hookeri Torr. P., 2517.

64. Microstylis unifolia (Michx.) BSP. A.

65. Spiranthes cernua (L.) Richard. \#

66. S. gracilis (Bigel.) Beck. A.

\section{Salicacece.}

67. Populus grandidentata Michx. A.

68. P. tremuloides Michx. E., 23 May, 1906.

69. Salix rostrata Richards. A.; 17 June, 1907.

XVI. Myricacece.

70. Myrica asplenifolia L. W. Also E., 25 May, 1906.

71. M. carolinensis Mill. W.

72. M. Gale L. W.

XVII. Betulacece.

73. Alnus incana (L.) Moench. A. Also K.

74. A. viridis DC. K. 
76. B. lenta L. \# Also K.

77. B. lutea Michx. f. W. Also K.

78. B. populifolia Marsh. W. Alșo E., 28 May, 1906.

Carpinus caroliniana Walt. $\mathrm{K}$.

79. Ostrya virginica (Mill.) K. Koch. \#

XVIII. Fagacean

80. Fagus grandifolia Ehrh. \#

81. Quercus alba L. W. H. Ropes, February, 1910.

82. Q. rubra L. W.

XIX. Urticacece.

83. Ulmus americana L. \#

XX. Polygonacece.

84. Polygonum cilinode Michx. W. Also K.

85. Rumex Acetosella L. \#

XXI. Nymphosacece.

86. Castalia odorata (Ait.) Woodville \& Wood. \#

XXII. Ranunculacece.

87. Anemone virginiana L. E., 1 August, 1909.

Cimicifuga americana Michx. $\mathrm{K}$.

Clematis virginiana $\mathrm{L}$. K.

88. Coptis trifolia (L.) Salisb. A.

89. Ranunculus acris L. M. A. Coe, July, 1909.

99. Thalictrum polygamum Muhl. \#

Zanthorhiza apiifolia L'Hér. K.

Magnoliacece.

Liriodendron Tulipifera L. K.

Magnolia acuminata L. K.

M. Fraseri Walt. K.

M. tripetala L. K.

M. virginiana L. K.

Calycanthacexe.

Calycanthus floridus L. K.

Berberidacece.

Berberis canadensis Mill. $\mathrm{K}$. 
XXIII. Fumariacee.

91. Corydalis sempervirens (L.) Pers. W.

XXIIIA. Cruciferc.

92. Brassica campestris L. E., 15 June, 1907.

XXIV. Saxifragacece.

Itea virginica $\mathrm{L}$. $\mathrm{K}$.

93. Ribes prostratum L'Hér. A.

XXV. Hamamelidacece.

94. Hamamelis virginiana $\mathrm{L}$. W.

\section{Platanacexe.}

Platanus occidentalis L. K.

XXVI. Rosacea.

95. Amelanchier canadensis (L.) Medic. W. Also E., 21-28 May, 1906.

96. A. spicata (Lam.) K. Koch. W. Also E., 21 May, 1906.

97. Fragaria vesca L., var. americana Porter. \#

98. Potentilla monspeliensis L. W.

99. Prunus pennsylvanica L. f. E., 25 May, 1906.

100. P. serotina Ehrh. \#

101. Pyrus melanocarpa (Michx.) Willd. A.

102. Rosa blanda Ait. M. A. Coe, July, 1909.

R. setigera Michx. K.

103. Rubus allegुheniensis Porter. A.

104. R. hispidus L. M. A. Coe, July, 1909.

105. R. idæus L., var. aculeatissimus [C. A. Mey.] Regel \& Tiling. A.

R. laciniatus Willd. K.

106. R. odoratus L. A.

107. Spiræa latifolia Borkh. W.

108. S. tomentosa L. \#

\section{Legruminosce.}

109. Apios tuberosa Moench. \#

Robinia hispida L. $\mathrm{K}$.

110. Trifolium agrarium $\mathrm{L}$. W.

111. T. pratense L. A.

112. T. repens L. W. 
XXVIII. Anacardiacece.

113. Rhus Toxicodendron L. W.

114. R. typhina L. W.

XXIX. Aquifoliacece.

Ilex glabra (L.) Gray. K.

I. monticola Gray. K.

115. I. verticillata (L.) Gray. Anonymously reported in July, 1906.

116. Nemopanthus mucronata (L.) Trel. E., 30 May, 1906.

XXX: Aceracece.

117. Acer pennsylvanicum L. A., 21 May, 1906.

118. A. rubrum L. A.

119. A. saccharum Marsh. \#

XXXI. Balsaminacece.

120. Impatiens biflora Walt.

XXXII. Vitacece.

121. Psedera vitacea (Knerr) Greene (?) \# P. sp. K.

122. Vitis æstivalis Michx. A.

XXXIII. Tiliacece.

123. Tilia americana $\mathrm{L}$. \#

\section{Hypericacece.}

Hypericum aureum Bart. $K$.

H. densiflorum Pursh. K.

124. H. perforatum L. M. A. Coe.

H. prolificum L. K.

125. H. virginicum L. A.

\section{Violacece.}

126. Viola blanda Willd. \#

127. V. pallens (Banks) Brainerd. \#

128. V. renifolia Gray. A.

129. V. septentrionalis Greene. (?) A.

XXXVI. Onagracece.

150. Epilobium angustifolium L. A.

131. E. densum Raf. \#

132. Enothera biennis L. "I" 
XXXVII. Araliacece.

133. Aralia hispida Vent. \#

134. A. nudicaulis L. A.

135. A. racemosa L. A.

\section{Umbelliferce.}

136. Sium cicutæfolium Schrank. P., no. 2521.

\section{Cornacea.}

Cornus alternifolia L. f. K.

137. C. canadensis L. W.

138. C. circinata L'Hér. A.

C. stolonifera Michx. K.

139. Nyssa sylvatica Marsh. \#

\section{Ericacece.}

140. Arctostaphylos Uva-ursi (L.) Spreng. A.

141. Chamædaphne calyculata (L.) Moench. E., May, 1906.

142. Chimaphila umbellata (L.) Nutt. A.

Clethra acuminata Michx. $\mathrm{K}$.

C. alnifolia L. K.

143. Gaultheria procumbens L. W.

144. Gaylussacia baccata (Wang.) K. Koch. A.

145. G. baccata (Warg.) K. Koch, f. glaucocarpa (Robinson) Mackenzie. \#

146. Kalmia angustifolia $\mathrm{L}$. W.

K. latifolia L. K.

Leiophyllum buxifolium (Berg.) Ell. K.

Leucothoë Catesbæi (Walt.) Gray. K.

147. Lyonia ligustrina (L.) DC. \#

148. Monotropa Hypopitys L. \#

149. M. uniflora L. W.

Oxydendrum arboreum (L.) DC. K.

150. Pyrola chlorantha Sw. W.

151. P. elliptica Nutt. W.

152. P. secunda L. W.

Rhododendron arborescens (Pursh.) Torr. K.

R. calendulaceum (Wilcox) Torr. K.

153. R. canadense (L.) BSP. W. E., 30 May, 1906. Also K.

R. catawbiense Michx. $K$.

R. maximum L. K. 
274 LIST OF PLANTS ON THREE MILE ISLAND。

R. Vaseyi Gray. K.

R. viscosum (L.) Torr. K.

154. Vaccinium corymbosum L. \#

155. V. pennsylvanicum Lam. A.

156. V. vacillans Kalm. \#

Diapensiacece.

Galax aphylla L. K.

Shortia galacifolia T. \& G. K.

XLI. Primulacece.

157. Lysimachia terrestris (L.) BSP. \#

158. Trientalis americana (Pers.) Pursh. A., 23 May, 1906.

\section{Styracacece.}

Halesia carolina L. K.

XLII. Oleacece.

159. Fraxinus americana L. \#

\section{Gentianacece.}

160. Gentiana Andrewsii Griseb. Anonymously reported, July, 1906. 161. Nymphoides lacunosum (Vent.) Fernald. \#

\section{Apocynacece.}

162. Apocynum androsæmifolium L. W.

\section{Asclepiadacece.}

163. Asclepias phytolaccoides Pursh. \#

\section{Labiatce.}

164. Lycopus uniflorus Michx. \#

165. Mentha arvensis L., var. canadensis (L.) Briquet. \#

166. Prunella vulgaris $\mathrm{L}$. \#

167. Scutellaria galericulata L. Anonymously reported, July, 1906.

XLVII. Scrophulariacece.

168. Gratiola aurea Muhl. \#

169. Melampyrum lineare Lam. W.

170. Verbascum Thapsus L. \# 
XLVIII. Orobanchacece.

171. Epifagus virginiana (L.) Bart. \#

172. Orobanche uniflora L. 17 July, 1907.

Bignoniacece.

Catalpa bignonioides Walt. $\mathrm{K}$.

\section{Plantaginacece.}

173. Plantago major L. \#

\section{Rubiacece.}

174. Cephalanthus occidentalis L. W. Also K.

175. Galium tinctorium L. W.

176. G. triflorum Michx. \#

177. Mitchella repens L. W.

\section{Caprifoliacece.}

178. Diervilla Lonicera Mill. A.

D. sessilifolia Buckley. K.

179. Lonicera canadensis Marsh. A.

L. japonica Thunb., var. Halliana Nicholson. K.

180. Viburnum acerifolium L. W.

181. V. alnifolium Marsh. A., 20 May, 1906.

182. V. cassinoides L. A.

183. V. dentatum L. W.

\section{Lobeliacex.}

184. Lobelia Dortmanna L. M. A. Coe, 12 August, 1906.

LIII. Composito.

186. Achillea Millefolium L. A.

187. Anaphalis margaritacea B. \& H. M. A. Coe, August, 1906.

188. Antennaria canadensis Greene. W.

189. A. fallax Greene. P., no. 2502.

190. A. neglecta Greene. \#

191. A. neodioica Greene. \#

192. A. plantaginifolia (L.) Richards. E., 1 June, 1909.

193. Aster acuminatus Michx. \#

194. A. divaricatus L. \#

195. A. dumosus L. \#

196. A. macrophyllus L., (or one of the varieties of this species). P. no. 2514. Also E., 1 June, 1909. 
276 LIST OF PLANTS ON THREE MILE ISLAND.

197. A novi-belgii L. \#

198. A. puniceus L. \#

199. A. umbellatus Mill. \#

200. A. undulatus L. P., no. 2518.

201. Bidens connata Muhl. (?) \#

202. Erigeron ramosus (Walt.) BSP. \#

203. Eupatorium perfoliatum L. \#

204. E. purpureum L. \#

205. Gnaphalium decurrens Ives. \#

206. Hieracium paniculatum L. M. A. Coe, August, 1906.

207. H. scabrum Michx. M. A. Coe, August, 1906.

208. Lactuca canadensis $\mathrm{L}$. \#

209. Prenanthes trifoliolata (Cass.) Fernald. M. A. Coe, August, 1906.

210. Solidago arguta Ait. P., no. 2523. \#

211. S. bicolor L. A.

212. S. cæsia L. \#

213. S. canadensis L. A.

214. S. graminifolia (L.) Salisb. \#

215. S. juncea Ait. A.

216. S. nemoralis Ait. A.

217. S. rugosa Mill. \#

218. Taraxacum officinale Weber. \# 